\title{
EXPONENTIAL CLOGGING TIME FOR A ONE DIMENSIONAL DLA
}

\author{
ITAI BENJAMINI AND CHRISTOPHER HOFFMAN
}

\begin{abstract}
In this paper a simple DLA type model is analyzed. In 1 the standard DLA model from [2] was considered on a cylinder and the arm growing phenomena was established, provided that the section of the cylinder has sufficiently fast mixing rate. When considering DLA on a cylinder it is natural to ask how many particles it takes to clog the cylinder, e.g. modeling clogging of arteries. In this note we formulate a very simple DLA clogging model and establish an exponential lower bound on the number of particles arriving before clogging appears. In particular we possibly shed some light on why it takes so long to reach the bypass operation.
\end{abstract}

\section{INTRODUCTION}

We start with an informal description of our model. Fix some $N \in \mathbb{N}$. Initially there is a particle only at the vertices 0 and $1 \in \mathbb{Z}$. A third particle performs a simple random walk started at positive infinity until at some random time when it stops and never moves again. If the particle is at a vertex $i$, it "freezes" there (stays there and remains there for all time) with probability equal to the number of particles at vertex $i-1$ divided by $N$. If the particle does not freeze at $i$ then it takes a step in simple random walk. The particle repeats this procedure of either freezing or taking one step of simple random walk until it freezes. When the third particle freezes, the forth particle starts and so on. By the analysis in [1] the cluster of the particles will grow to the right leaving typically only $o(N)$ particles at each site, thus it will become harder for particles to penetrate deep beyond the arm to completely fill a site with $N$ particles. Thus the time required before there is one site with $N$ particles is at least exponential in $N$. This is the main result of this note.

\section{Formal Definition of the process}

We will inductively define the following random variables. The variables $\{w(j, t)\}_{j, t \in \mathbb{N}}$ give the location of the $j$ th particle after it has take $t$ steps. The random variables $\{w(j, \infty)\}_{j \in \mathbb{N}}$ indicate where the $j$ th particle freezes. And the variables $\{f(k, i)\}_{k, i \in \mathbb{N}}$ indicate the number of particles labeled less than or equal to $i$ which have frozen at position $k$.

We define these variables as follows. Let $w(1, t)=0$ for all $t$. For a fixed $j$ assume we have defined the $w(j, t)$ in such a way that for all $j^{\prime} \leq j$ we have that $\lim _{t \rightarrow \infty} w(j, t)$ exists and that we have defined $f(k, i)$ for all $i<j$. Then we define

$$
w(j, \infty)=\lim _{t \rightarrow \infty} w(j, t)
$$

and

$$
f(k, i)=\left|\left\{i^{\prime} \leq i: w\left(i^{\prime}, \infty\right)=k\right\}\right| .
$$

For any $j>1$ let $w(j, 1)=j+2$ and $w(j, 2)=j+1$.

Date: November 1, 2018. 
For any $j>1$ and $t>2$ if $w(j, t)=w(j, t-1)$ then define $w(j, t+1)=w(j, t)$. If $w(j, t) \neq w(j, t-1)$ then set

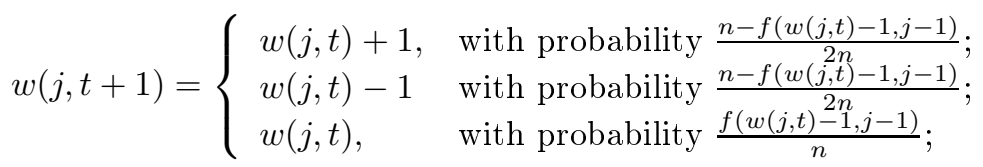

As simple random walk on $\mathbb{Z}$ is recurrent we have that $w(j, t)=w(j, t+1)$ for some $t$ almost surely. Thus all of the random variables are well defined almost surely.

If $w(j, \infty)=k$ then we say that particle $j$ freezes at $k$. If for some $j$ there exists a $t$ with

$$
k=w(j, t)=w(j, t+1)<\min _{t^{\prime}<t} w(j, t)
$$

then $w(j, \infty)=k$ and we say that particle $j$ freezes upon arrival at $k$. If for some $k$ there exists $t$ with $f(k, t)=n$ then we say that there is a blockage at $k$. Define the random variable

$$
B=\inf \{k: \text { there exists a blockage at } k\} .
$$

Thus $B$ indicates the position of the leftmost blockage.

Theorem 2.1. There exist $c>0$ such that

$$
\mathbf{P}\left(B<e^{c n}\right)<e^{-c n} .
$$

We make the following comments about our theorem.

- The proof given below clearly works for directed random walks or, more generally, any nearest neighbor process on $\mathbb{Z}$. We only need to modify the model so that the particle either freezes at some location or disappears off to infinity.

- An easy upper bound on $B$ is that for any $\epsilon>0$ there exists $c$ such that $\mathbf{P}\left(B>c N^{N}\right)<\epsilon$. It is of interest to get the exact order of $B$.

- The question from [1] regarding clogging of a cylinder $G \times \mathbb{Z}$ is more complicated due to the geometry of the possible cuts. Finding the distribution on the location of the leftmost clogging in the cylinder is an interesting open question.

\section{Proof}

For any $k$ let $S(k)$ be the event that there exists $i$ with $f(k, i)=n$.

Lemma 3.1. There exists $c>0$ such that for any $k$ and $n$

$$
\mathbf{P}(S(k)) \leq e^{-c n} .
$$

Proof. Fix $k$. For any $k^{\prime}, J$ and $J^{\prime}$ let $C\left(k^{\prime}, J, J^{\prime}\right)$ be the number of $j$ such that $J<j \leq J^{\prime}$ and $\min _{t} w(j, t) \leq k^{\prime}$. Let $I_{n / 2}\left(I_{3 n / 4}, I_{7 n / 8}, I_{15 n / 16}\right)$ be the minimal value such that $f\left(k, I_{n / 2}\right)=n / 2(3 n / 4,7 n / 8,15 n / 16$ respectively) if such a value exists. If $I_{n / 2}$ (or any of $I_{3 n / 4}, I_{7 n / 8}$, and $I_{15 n / 16}$ ) is undefined then $S(k)$ does not occur.

Finally let

$$
g(i)=\mid\left\{j \in\left(I_{n / 2}, i\right): \text { particle } j \text { freezes upon arrival at } k+1\right\} \mid .
$$


Note that for all $i>I_{n / 2}$

$$
f(k, i)) \leq n / 2+C\left(k, I_{n / 2}, i\right)
$$

and

$$
f(k+1, i)) \geq g(i)
$$

Thus if the event $S(k)$ occurs then there must be some $i$ such that $C\left(k, I_{n / 2}, i\right) \geq$ $n / 2$ and $g(i)<n$.

If $S(k)$ occurs then one of the following three things must happen:

(1) $g\left(I_{3 n / 4}\right)<.22 n$,

(2) $g\left(I_{7 n / 8}\right)<.57 n$, or

(3) $g\left(I_{15 n / 16}\right)<n$.

For every $j \in\left[I_{n / 2}, I_{3 n / 4}\right]$ with $\min _{t} w(j, t) \leq k+1$ the probability that the particle freezes upon arrival at $k+1$ is at least one half. Thus if the first event occurs of the first $.47 n$ particles to arrive at $k+1$ less than forty seven percent of them freeze upon arrival at $k+1$. The probability of this is decreasing exponentially in $n$.

For every $j \in\left[I_{3 n / 4}, I_{7 n / 8}\right]$ with $\min _{t} w(j, t) \leq k+1$ the probability that the particle freezes upon arrival at $k+1$ is at least .75 . Thus if the first event does not occur but the second event does, then of the first $.475 n$ particles to arrive at $k+1$ after $I_{3 n / 4}$ less than 74 per cent of them freeze upon arrival at $k+1$. The conditional probability of this given the compliment of the first event is decreasing exponentially in $n$.

For every $j \in\left[I_{7 n / 8}, I_{15 n / 16}\right]$ with $\min _{t} w(j, t) \leq k+1$ the probability that the particle freezes upon arrival at $k+1$ is at least .875 . Thus if the first event does not occur but the second event does, then of the first $.4925 n$ particles to arrive at $k+1$ after $I_{3 n / 4}$ less than 87.4 per cent of them freeze upon arrival at $k+1$. The conditional probability of this given the compliment of the second event is decreasing exponentially in $n$.

Thus the probability that there exists $i$ such that $C\left(k, I_{n / 2}, i\right) \geq n / 2$ and $g(i)<n$ is exponentially small in $n$. Thus the probability of $S(k)$ is as well.

Proof of Theorem 2.1. This follows by replacing $c$ with $c / 2$ in Lemma 3.1,

\section{REFERENCES}

[1] I. Benjamini, A. Yadin, Diffusion Limited Aggregation on a Cylinder. Comm. Math. Phys. To appear (2007) arXiv:math/0701201.

[2] T. Witten, L. Sander Diffusion-limited aggregation. Phys. Rev. B 27, 5686 - 5697 (1983).

Department of Mathematics, The Weizmann Institute, Rehovot, Israel 76100 and Microsoft Research

E-mail address: itai.benjamini@weizmann.ac.il

Department of Mathematics, University of Washington, Seattle, Wa 98195

E-mail address: hoffman@math.washington.edu 